DOI: https://doi.org/10.46296/ig.v3i6.0016

\title{
CINÉTICA DE DESHIDRATACIÓN DE LA UVA (VITIS VINIFERA L.)
}

\section{DEHYDRATION KINETICS OF GRAPE (VITIS VINIFERA L.)}

\author{
Bazurto-Vera Karol ${ }^{1 *}$; Cevallos-Cedeño María ${ }^{2}$; Vilcacundo-Alcívar Anlly ${ }^{3}$ \\ ${ }^{1}$ Universidad Técnica de Manabí, UTM. Portoviejo, Ecuador. \\ ¿Universidad Técnica de Manabí, UTM. Portoviejo, Ecuador. \\ 3Universidad Técnica de Manabí, UTM. Portoviejo, Ecuador.
}

*Correo: karolbazurto@gmail.com

\begin{abstract}
Resumen
El presente trabajo se fundamenta en el proceso de deshidratación de la uva (Vitis vinifera L.), puesto que el proceso de secado es de gran importancia en diversos procesos químicos industriales, ya que permite alargar la vida útil de cualquier alimento, obteniéndose productos con mayor valor agregado. El objetivo de esta investigación fue evaluar la cinética de deshidratación de la uva a partir de la curva de velocidad contra la humedad media y la curva de humedad total contra el tiempo. El contenido de humedad en la uva se determinó por el método de secado por estufa de aire caliente forzado, basándose en la pérdida de peso de la uva en relación al tiempo, la muestra desde el tiempo cero se pesó y cada 5 minutos durante 1 hora y 25 minutos, se realizó el procedimiento a una temperatura de $60^{\circ} \mathrm{C}$. Al obtener los datos de cada peso, siendo un total de 15 datos, se realizó los procedimientos para calcular la humedad total, humedad media, humedad libre y la velocidad crítica de secado. El contenido de agua en la uva tuvo un valor final de $7,02 \times 10^{-5} \mathrm{~kg}$ de agua, con una velocidad de secado de $0,095951332 \mathrm{Kg} / \mathrm{h}$ y una humedad media de $0,0001016 \mathrm{KgH}_{2} \mathrm{O} / \mathrm{Kg}$ sólido seco. Se concluye que la pérdida de peso de la uva es directamente proporcional al tiempo y a la temperatura a la que fue sometida la muestra.
\end{abstract}

Palabras clave: deshidratación, uva, tiempo, humedad, temperatura.

\begin{abstract}
The present work is based on the dehydration process of the grape Vitis vinifera L.), this drying process is of great importance since it allows to extend the useful life of any food, obtaining products with higher added value. The objective of this investigation was to evaluate the dehydration kinetics of the grapes from the speed curve against the average humidity and the curve of total humidity against time. The moisture content in the grapes is determined by the forced hot air drying method, the limitations in the weight loss of the grapes in relation to time, the sample from time zero was weighed and every 5 minutes for 1 hour and 25 minutes, the procedure was performed at a temperature of $60^{\circ} \mathrm{C}$. When obtaining the data for each weight, for a total of 15 data, the procedures were performed to calculate the total humidity, average humidity, free humidity and the critical speed of drying. The water content in the grape had a final value of $7.02 \times 10^{-5} \mathrm{~kg}$ of water, with a drying speed of $0.095951332 \mathrm{Kg} / \mathrm{h}$ and an average humidity of $0.0001016 \mathrm{KgH}_{2} \mathrm{O} / \mathrm{Kg}$ dry solid. This reflects that the weight loss of the grape is directly proportional to the time and temperature that the sample was sometimes.
\end{abstract}

Keywords: dehydration, grape, time, humidity, temperature.

Información del manuscrito:

Fecha de recepción: 30 de abril de 2020

Fecha de aceptación: 22 de junio de 2020

Fecha de publicación: 10 de julio de 2020 


\section{Introducción}

La uva es una fruta proveniente de la vid o parra, llamada científicamente como Vitis Vinifera L., se define como un alimento saludable no solamente por su valor nutritivo, sino también por sus componentes fenólicos con potente acción antioxidante y además, ayuda en la mejora de la salud de los pacientes con diabetes tipo II (Kanellos, 2014).

Las frutas además contienen una alta proporción de agua y la distribución de fuentes potenciales de los compuestos bioactivos, antioxidantes, minerales, micronutrientes, entre otros (El Anany, 2015). La uva presenta un contenido de agua de aproximadamente $75-85 \%$ y de sólidos solubles de 15-25\% (Hidalgo et al., 2016).

La estabilidad de las frutas, como de cualquier alimento se ve fuertemente afectada por los procesos de almacenamiento. La deshidratación permite alargar su vida útil (debido a que es perecedera y susceptible de diversas alteraciones microbiológicas y bioquímicas), también permite la reducción de peso y volumen de la misma, ahorrando de esta manera en transporte, almacenamiento y envasado, por otro lado, facilita la disponibilidad de este producto en toda la época del año, pero puede causar cambios que son perjudiciales en el color, sabor, valor nutricional y en el contenido de antioxidantes, dependiendo de la intensidad con la que se lleve a cabo el proceso de secado (Laborde et al., 2015).

La deshidratación por flujo de aire caliente, se origina a partir de la evaporación-eliminación del contenido de agua, por ende, impide el crecimiento bacteriano (SerpaGuerra et al., 2015; Vega-Gálvez et al., 2009). En el secado de frutas mediante este proceso se pueden afectar las propiedades sensoriales y el valor nutricional si se almacena a temperaturas muy altas. La temperatura de secado es una variable fundamental en los estudios cinéticos de cualquiera de los procesos (García-Pereira et al., 2013).

\section{Metodología}

La investigación fue descriptivaexperimental con un enfoque cuantitativo. Los procedimientos de secado fueron realizados por estufa y se obtuvieron los resultados de 
humedad total, media, libre $y$ velocidad crítica de secado, a través de los pesos que se obtuvieron con respecto al tiempo y temperatura a la que fue sometida la muestra, el proyecto experimental fue realizado en el Laboratorio de Microbiología de la Universidad Técnica de Manabí, Ecuador.

\subsection{Recepción de la muestra}

Las uvas fueron obtenidas en un mercado local del cantón Portoviejo situado en la provincia de Manabí, Ecuador

(1³'16.49"S $\left.80^{\circ} 27^{\prime} 16.02 " \mathrm{O}\right)$; estas frutas fueron seleccionadas y lavadas con el fin de eliminar algún residuo que contengan.

\subsection{Secado}

El proceso de secado o deshidratación de la uva se llevó a cabo por medio de la estufa de circulación de aire caliente forzado Memmert UFE, la cual consiste en la pérdida de peso de la muestra por evaporación del agua.

El principio operacional del método de secado por estufa se basa en la determinación de humedad utilizando estufa y balanza analítica, que incluye la preparación de la muestra, pesado, secado, enfriado y pesado nuevamente de la muestra (Nollet, 1996).

De acuerdo a Laborde et al., (2015), en su trabajo sobre las pasas de uva de bajas calorías obtenidas por deshidratación combinada, sometieron a la uva a una temperatura de $60^{\circ} \mathrm{C}$, mostrando resultados que demuestran la obtención de uva deshidratada saludable con bajo contenido calórico por sustitución de los azúcares propios de la fruta por un edulcorante natural sin calorías, mediante la optimización de métodos combinados de la deshidratación, manteniendo adecuadamente el contenido de polifenoles totales, aunque con una reducción de la eficiencia antioxidante de la fruta fresca, por lo tanto, en la presente investigación se ha colocado la muestra en la estufa a $60^{\circ} \mathrm{C}$, teniendo referencia que ya se ha deshidratado la uva a esta temperatura modificando la variable de tiempo. El procedimiento permitió medir las variaciones en el peso de la muestra respecto al tiempo, hasta que el peso de la muestra permanezca constante.

El procedimiento consistió en un crisol en el que se colocó la muestra, 
se pesó en la balanza analítica de precisión de 3200G modelo EJ3202A, para determinar su peso inicial, luego fue llevado a la estufa de secado Memmert UFE. Cada 5 minutos se realizó el procedimiento de pesado desde el tiempo inicial a una temperatura de $60^{\circ} \mathrm{C}$, se medía el peso y la diferencia de pérdida de peso, con un total de 15 datos.

\section{Resultados y discusión}

Los datos obtenidos durante la deshidratación de la uva permitieron determinar la cinética de deshidratación de la fruta, en la cual se obtuvo la curva de velocidad contra la humedad media y la curva de humedad total contra el tiempo, durante 1 hora y 25 minutos a $60^{\circ} \mathrm{C}$.

A continuación, se expone una breve descripción del procedimiento de la aplicación de las ecuaciones empleadas para realizar los cálculos de los datos obtenidos para los cálculos de la humedad (total, media y libre) y la velocidad crítica de secado.

Nomenclatura:

Se mencionan a continuación la nomenclatura de las variables en las fórmulas aplicadas.
Y: Humedad total $\left(\mathrm{Kg} \mathrm{H}_{2} \mathrm{O}\right)$.

R: Velocidad crítica de secado $(\mathrm{Kg} / \mathrm{h})$

M1: peso de la uva con humedad (kg)

Ls: Sólido seco $(\mathrm{kg})$

A: Área de crisol $\left(0,007853982 \mathrm{~m}^{2}\right)$

$\Delta t=$ Diferencia del tiempo (h)

$\Delta X=$ Diferencia de la humedad libre

$$
\left(\frac{k g H 2 O}{K g \text { sólido seco }}\right)
$$

Para el cálculo se utilizó un peso final de la uva seca de 0,0077899 kg.

Se realizó la diferencia de pesos, siendo estos el inicial y el final, así sucesivamente con cada peso restando $0,0077899 \mathrm{~kg}$ de uva seca, para obtener la humedad total de la muestra de uva.

$Y=M 1-L s=(0,0082753-0,0077899)$ $=0,0004854 \mathrm{~kg} \mathrm{H}_{2} \mathrm{O}$

Mientras que la humedad libre, se basó en el resultado de la humedad libre entre el peso final de la uva seca.

Humedad libre $=\frac{Y}{L s}$

Humedad media $=\frac{M 1+M 2}{2}$

$R=-\frac{L s}{A} \frac{\Delta \mathrm{X}}{\Delta \mathrm{t}}=$ 
Los resultados, se realizaron de igual manera, reemplazando las variables de peso de la uva con humedad y tiempo, para obtener la humedad total, libre, media y velocidad critica de secado a partir de las fórmulas planteadas.

Se obtuvieron los resultados de peso expresados en kilogramos, mientras que el tiempo en minutos fue calculado y convertido en horas de la deshidratación (secado) a través de la estufa por medio de aire caliente forzado, cada 5 minutos se pesó la muestra a una temperatura de $60^{\circ} \mathrm{C}$, con un total de 15 datos hasta obtener un peso constante de la uva.

Tabla 1.

Datos obtenidos en el secado de la uva

\begin{tabular}{|c|c|c|}
\hline $\mathbf{N}^{\circ}$ & Tiempo (hora) & Peso uva (kg) \\
\hline 0 & 0 & 0,0082753 \\
\hline 1 & 0,08333333 & 0,0082585 \\
\hline 2 & 0,16666667 & 0,0082506 \\
\hline 3 & 0,25 & 0,0082389 \\
\hline 4 & 0,33333333 & 0,0082287 \\
\hline 5 & 0,41666667 & 0,0082023 \\
\hline 6 & 0,5 & 0,008183 \\
\hline 7 & 0,58333333 & 0,008158 \\
\hline 8 & 0,66666667 & 0,0081195 \\
\hline 9 & 0,75 & 0,0080866 \\
\hline 10 & 083333333 & 0,0080588 \\
\hline 11 & 0,91666667 & 0,0080294 \\
\hline 12 & 1 & 0,0080022 \\
\hline 13 & 1,08333333 & 0,0079782 \\
\hline 14 & 1,16666667 & 0,0079229 \\
\hline 15 & 1,25 & 0,0078601 \\
\hline & & \\
\hline
\end{tabular}


Tabla 2.

Valores de humedad total, humedad libre, humedad media y velocidad crítica respecto al tiempo

\begin{tabular}{|c|l|c|c|c|}
\hline $\mathbf{N}^{\circ}$ & H. total & H. libre & H. media & $\begin{array}{c}\text { Velocidad } \\
\text { critica }\end{array}$ \\
\hline 0 & 0,0004854 & 0,062311455 & & \\
\hline 1 & 0,0004686 & 0,060154816 & 0,000477 & 0,025668509 \\
\hline 2 & 0,0004607 & 0,059140682 & 0,00046465 & 0,012070311 \\
\hline 3 & 0,000449 & 0,057638737 & 0,00045485 & 0,017876283 \\
\hline 4 & 0,0004388 & 0,05632935 & 0,0004439 & 0,015584452 \\
\hline 5 & 0,0004124 & 0,052940346 & 0,0004256 & 0,040336229 \\
\hline 6 & 0,0003931 & 0,050462779 & 0,00040275 & 0,029488228 \\
\hline 7 & 0,0003681 & 0,047253495 & 0,0003806 & 0,038197186 \\
\hline 8 & 0,0003296 & 0,042311198 & 0,00034885 & 0,058823667 \\
\hline 9 & 0,0002967 & 0,03808778 & 0,00031315 & 0,050267497 \\
\hline 10 & 0,0002689 & 0,034519057 & 0,0002828 & 0,042475271 \\
\hline 11 & 0,0002395 & 0,030744939 & 0,0002542 & 0,044919891 \\
\hline 12 & 0,0002123 & 0,027253238 & 0,0002259 & 0,041558539 \\
\hline 13 & 0,0001883 & 0,024172326 & 0,0002003 & 0,036669299 \\
\hline 14 & 0,000133 & 0,01707339 & 0,00016065 & 0,084492176 \\
\hline 15 & $7,02 \mathrm{E}-05$ & 0,009011669 & 0,0001016 & 0,095951332 \\
\hline
\end{tabular}

*H. total: $\left(\mathrm{Kg} \mathrm{H}_{2} \mathrm{O}\right)$, humedad libre $\left(\mathrm{Kg} \mathrm{H}_{2} \mathrm{O} / \mathrm{Kg} \mathrm{S} . \mathrm{S}\right)$, humedad media $\left(\mathrm{KgH}_{2} \mathrm{O} / \mathrm{Kg} \mathrm{S} . \mathrm{S}\right)$, velocidad crítica del secado $(\mathrm{R})(\mathrm{Kg} / \mathrm{h})$.

A continuación, se presenta la figura respecto al tiempo de secado de la

1, que refleja la gráfica obtenida con uva.

los datos de humedad total con

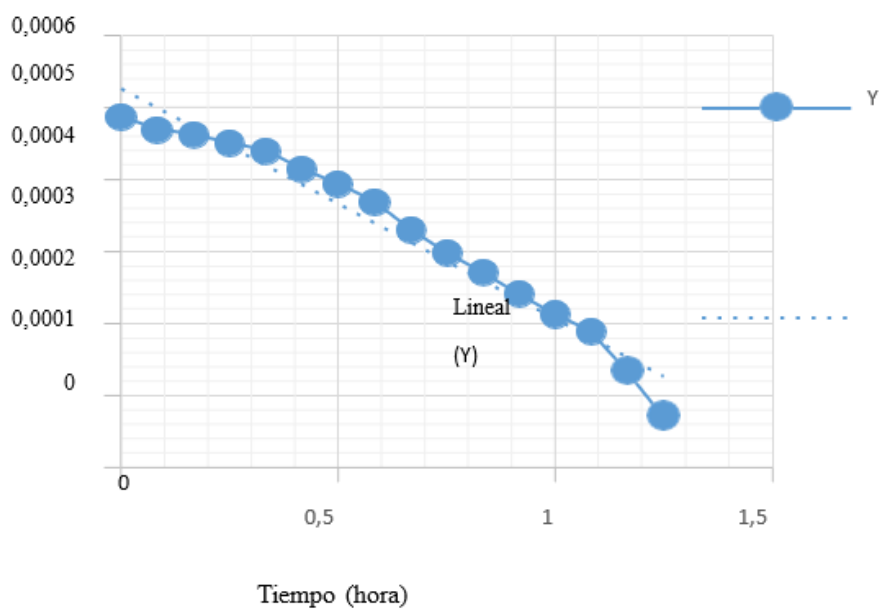

Figura 1. Gráfica de los datos de humedad total vs tiempo 


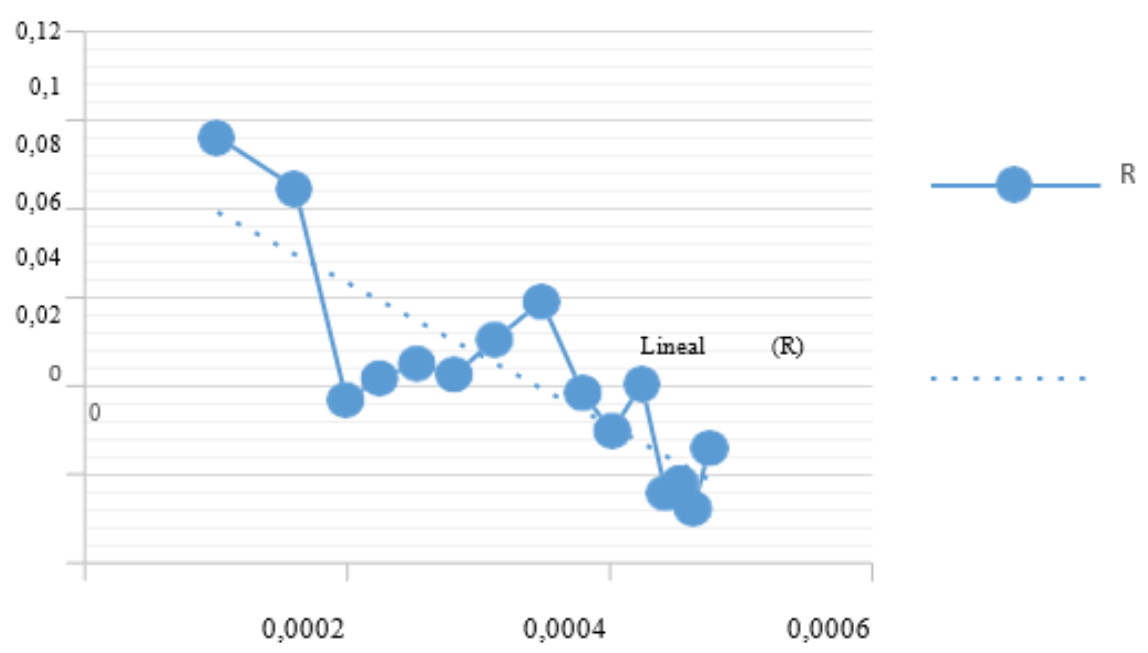

Figura 2. Gráfica de los datos de velocidad crítica del secado vs humedad media

Las ecuaciones de ajuste de cada gráfica son las siguientes:

Para la gráfica 1:

$$
\begin{aligned}
& y=-0,0003 x+0,0005 \\
& R^{2}=0,9633
\end{aligned}
$$

Para la gráfica 2:

$y=-160,85 x+0,0952$

$R^{2}=0,6784$

Los resultados obtenidos en la tabla 1 muestran los valores experimentales del tiempo en horas y el peso de la uva en kilogramos, los cuales sirven para calcular la humedad total, libre, media y la velocidad critica de secado.

La humedad libre inicial en la uva es de $0,062311455 \mathrm{~kg} \mathrm{H}_{2} \mathrm{O} / \mathrm{Kg}$ sólido seco, que es la humedad que puede perder el sólido después de un tiempo de contacto con el aire en las condiciones dadas y constantes; en la tabla 2 se puede observar que al finalizar la operación de secado se obtuvo una humedad libre de 0,0090116669 kg $\mathrm{H}_{2} \mathrm{O} / \mathrm{Kg}$ sólido seco. Esta tabla también evidencia como a medida que aumenta el tiempo, la cantidad de humedad libre que se va eliminando va disminuyendo, lo cual es explicado por las etapas de la curva de secado.

La desviación estándar de estos datos es 0,0161 por lo que los valores individuales no difieren tanto de la media que es 0,0418.

La humedad media es un promedio obtenido entre los valores de la humedad libre, siendo el valor inicial 0,000477 y el final 0,0001016 $(\mathrm{Kg}$ 
$\mathrm{H}_{2} \mathrm{O} / \mathrm{Kg}$ sólido seco) con una desviación estándar de los datos de 0,0001162 con respecto a la media de 0,00032912 .

En la Figura 2 como en la tabla 2, se representa la velocidad crítica del secado con respecto a la humedad media; estos datos demuestran que a medida que aumenta la velocidad de secado la humedad media presente en la uva va disminuyendo, se observa que inicialmente la velocidad es de 0,025668509 Kg/h y la humedad media de 0,000477 $\mathrm{KgH}_{2} \mathrm{O} / \mathrm{Kg}$ sólido seco y como valores finales se obtuvo una velocidad de $0,095951332 \mathrm{Kg} / \mathrm{h}$ y la humedad media de 0,0001016 $\mathrm{KgH}_{2} \mathrm{O} / \mathrm{Kg}$ sólido seco, por lo que se puede evidenciar que a medida que aumenta la velocidad de secado es más complicado eliminar mayores cantidades de agua presente en la uva.

Se observa en la gráfica 1 de la humedad total con respecto al tiempo, que a medida que avanza el tiempo la humedad presente en la uva va disminuyendo, logrando obtener al final un valor de $7,02 \times 10^{-5}$ kg de agua en la uva; por lo tanto, para poder eliminar toda la humedad $(75-85 \%)$ se debe continuar el secado de la uva por un tiempo aproximado de 24 horas a una temperatura de $60^{\circ} \mathrm{C}$, ya que de esta forma se obtendría un valor constante del peso de la uva indicando que ya no se puede eliminar más agua.

Sin embargo, para un peso inicial de $0,0082753 \mathrm{~kg}$ uva la humedad presente es de 7,034×10-3 kg (85\%) por lo que aun faltaría por eliminar $6,964 \times 10-3 \mathrm{~kg}$ de agua de la uva.

En los ajustes de modelo para la gráfica 1 , se tiene un coeficiente de determinación de 0,9633 por lo que los datos se ajustan considerablemente mientras que para la gráfica 2 se debería cambiar el modelo lineal por otro ya que su coeficiente de determinación es bastante bajo siendo 0,6784; por lo que sería adecuado utilizar otros estadísticos para encontrar el mejor modelo para este grupo de datos.

De los resultados obtenidos se deduce que las curvas de encogimiento de las uvas son dependientes de la velocidad del aire de secado, si bien es cierto que estas diferencias se incrementan a medida que avanza el proceso. 


\section{Conclusiones}

Se obtuvieron cinéticas de secado de la uva en diferentes tiempos a una de temperatura de $60^{\circ} \mathrm{C}$, las cuales son humedad total con respecto al tiempo, y la de velocidad de secado con respecto a la humedad media.

Los factores de operación de temperatura, tiempo y velocidad de secado tienen un efecto importante sobre el proceso de encogimiento 0 deshidratación de la uva. Siendo el factor tiempo el más relevante.

Las cantidades de agua eliminadas en cada lapso de tiempo, fueron muy bajas, iniciando con una velocidad de secado baja de $0,0256 \mathrm{~kg} / \mathrm{h}$ y terminando a los 75 minutos con una velocidad de 0,0959 kg/h. Resultando que la velocidad de secado es inversamente proporcional a la humedad total.

\section{Bibliografía}

El Anany, A. M. (2015). Nutritional composition, antinutritional factors, bioactive compounds and antioxidant activity of guava seeds (Psidium Myrtaceae) as affected by roasting processes. Journal of Food Science and Technology, 52(4), 21752183. DOI: $10.1007 / \mathrm{s} 13197-$ 013-1242-1
García-Pereira, A., Muñiz-Becerá, S., Hernández-Gómez, A., González, L. M., \& Fernández-Valdés, D. (2013). Análisis comparativo de la cinética de deshidratación Osmótica y por Flujo de Aire Caliente de la Piña (Ananas Comosus, variedad Cayena lisa). Revista Ciencias Técnicas Agropecuarias, 22(1), 62-69.

Hidalgo, R., Gómez, M., Rojas, P., Soliz, M., Soliz, R., Quiroga, D., Saravia, G., y Saavedra, D. (2016). Propiedades medicinales de la semilla de uva. Revista de investigación e información de salud, 11(26), 53-57.

Kanellos, P. T., Kaliora, A. C., Tentolouris, N. K., Argiana, V., Perrea, D., Kalogeropoulos, N., ... \& Karathanos, V. T. (2014). A pilot, randomized controlled trial to examine the health outcomes of raisin consumption in patients with diabetes. Nutrition, 30(3), 358-364.

Laborde, M., Barreto, G., \& Pagano, A. (2015). Pasas de uva de bajas calorías obtenidas por deshidratación combinada: optimización del proceso y evaluación de la eficiencia antioxidante. Avances en Ciencias e Ingeniería, 6(1), 17-30.

Nollet, L. (1996). Handbook of food analysis. M. Dekker, New York. 
Serpa Guerra, A. M., Vásquez Osorio, D. C., Castrillón Martínez, D. C., \& Hincapié Llanos, G. A. (2015). Comparison of two dehydration techniques for" pear" guava (Psidium guajava I.) on the effects of the vitamin C content and on the behavior of the technical and functional properties of the dietary fiber. Revista Lasallista de Investigación, 12(1), 10-20.
Vega-Gálvez, A., Di Scala, K., Rodríguez, K., LemusMondaca, R., Miranda, M., López, J., \& Perez-Won, M. (2009). Effect of air-drying temperature on physicochemical properties, antioxidant capacity, colour and total phenolic content of red pepper (Capsicum annuum, L. var. Hungarian). Food Chemistry, 117(4), 647653.

DOI: 10.1016/j.foodchem.2009.04. 066 